\title{
Black esophagus due to metastasis colonic carcinoma
}

\begin{abstract}
The black esophagus (BE) is endoscopically seen as diffuse, circumferential, black lesion usually in distal one -third of esophagus and extension to proximal esophagus is common. The First case was reported in 1990 by Goldenberg et al. Between 1965 and 2006, 88 patients with BE have been reported. Although the etiology is poorly recognized, tissue insult in BE reportedly results from hypo-perfusion, NG tube associated infection, and thromboembolic phenomenon from solid tumors or hematological malignancy. Here, we report a 79years old with metastasis colorectal cancer presented with black esophagus.

This is a 79years old chronic smoker male presented to our institution with unintentional weight loss about 40 pounds and constipation. During the work up of weight loss, CT was performed and revealed the colonic neoplasm with liver, lung and osseous lesions and the right portal vein thrombosis. Patient subsequently underwent colonoscopy which revealed a large, nearly obstructing infiltrative carcinoma at recto-sigmoid junction. Due to his presentation of history of painless, persistent, progressive odynophagia and loss of appetite, EGD was performed and showed LA class D esophagitis overlying blackish exudate seen at $25 \mathrm{~cm}$ from incisor extending up to $40 \mathrm{~cm}$ (Figure 1). Pathological report from esophagus showed squamous esophageal mucosa with mild acute nonspecific esophagitis (Figure 2) but no fungus detected by PAS stain. Further treatment options were offered to the patient, however the patient and family chose to continue hospice care and conservative management.

Black esophagus (BE) is rare but increasing incidence in older men with upper GI symptoms. In case report, presumed mechanism is likely due to vascular insult with its provoked thromboembolic phenomenon. Management of BE should emphasize on improving perfusion, treating comorbidities and associated infection if present, and reduce further tissue injury using proton pump inhibitors to prevent complications.
\end{abstract}

\author{
Volume 7 Issue 4 - 2017
}

\begin{abstract}
Kyawzaw Lin,' Aung Naing Lin,' Sithu Lin,' Thinzar Lin,' Sandar Linn, ${ }^{2}$ Ying Xian Liu ${ }^{3}$

'Internal Medicine Department, The Brooklyn Hospital Center, USA

${ }^{2}$ Gastroenterology fellow, The Brooklyn Hospital Center, USA ${ }^{3}$ Attending Pathologist, The Brooklyn Hospital Center, USA
\end{abstract}

Correspondence: Kyawzaw Lin MD, Department of Medicine, The Brooklyn Hospital Center, 1733 West Ist Street, Brooklyn, NY I I223, USA, Tel 315-664-1916,

Email dr.kyawzawlin2015@gmail.com

Received: October 5, 2017 | Published: October II, 2017
Abbreviations: BE, black esophagus; EGD, esophagogastroduodenoscopy; NG tube, nasogastric tube; CT scan, computed tomography; CEA, carcinoembryonic antigen; CA, cancer antigen; AEN, acute esophageal necrosis

\section{Introduction}

Gurvits syndrome is rare but a unique syndrome presenting with acute esophageal necrosis or the black esophagus and is found in critical settings associated with diabetes mellitus, malignancy, gastroesophageal reflux disease or terminally ill patients. The overall mortality rate is reported to be $31.8 \%$ in the literature related to underlying medical conditions but death due to esophageal necrosis is less than $6 \%$ of all cases. ${ }^{1}$ The prevalence of the black esophagus is $0.05 \% .5$ in 9,179 EGD performed from 2005-2007 in retrospective chart analysis. ${ }^{2}$ The First case was reported in $1990 .{ }^{3}$ Only 88 cases of $\mathrm{BE}$ was reported in the literature in 40years. Although the etiology is poorly recognized, tissue insult in AEN results from hypo-perfusion, ischemia, chemical injury from lye ingestion, NG tube associated infection, severe vomiting, gastric outlet obstruction, anticardiolipin antibody syndrome, thromboembolic phenomenon from solid tumors or hematological malignancy, lactobacillus acidophilic infection, antibiotics including tetracycline and hypothermia. Here, we present one case of the black esophagus in 79year old chronic smoker concomitant with infiltrative adenocarcinoma of colon with distant metastases and portal vein thrombosis.

\section{Case presentation}

A 79year old chronic smoker (60 pack years and ex-smoker for 30years) male with no significant medical history was admitted for painless, persistent, progressive odynophagia for two weeks. He reported loss of appetite, change in voice, and unintentional loss of 40 pounds with loss of taste in two months associated with constipation. He denies nausea, vomiting, abdominal pain, lower abdominal discomfort, chest pain, cough, palpitation, hematemesis, hemoptysis or melena. Physical examination was unremarkable except mild tenderness in left lower quadrant, no guarding or rigidity, bowel sounds heard in all four quadrants but no palpable organomegaly. Basic metabolic panel and Liver function test are within normal limit. CEA was 1162 and CA 19.9 was 5885.3. Abdomen X-ray showed moderate amount of stool in the colon and chest X-ray showed bilateral rounded opacities of uncertain etiology. Due to anemia work up and negative fecal occult blood, CT scan of abdomen with contrast was done and showed neoplasm of distal sigmoid colon and rectum with extension along inferior mesenteric artery, para-aortic, retroperitoneal, and pelvic lymphadenopathy, metastatic disease to the liver, lungs and bones, and thrombosis of the right portal vein. Later, EGD showed LA class D esophagitis seen at $25 \mathrm{~cm}$ from incisor extending up to $40 \mathrm{~cm}$. Overlying blackish exudate was seen at area of the esophagitis (Figure 1). Colonoscopy showed large, erythematous, near obstructing friable mass at the recto-sigmoid junction. Multiple biopsies were taken from the mass. In order to relieve lumen obstruction, metallic colonic stent 
was placed .Pathological report from Esophagus showed squamous esophageal mucosa with mild acute nonspecific esophagitis (Figure 2) (Figure 3) but no fungus detected by PAS stain. Antrum and body biopsy report showed mild chronic nonspecific gastritis. Rectosigmoidal biopsy showed Infiltrating adenocarcinoma (Figure 4). Surgery team was consulted but the patient is not a candidate for surgical procedure. Oncology was consulted for aggressive treatment but the patient and family appreciated palliative care and he is on enoxaparin for portal vein thrombosis.

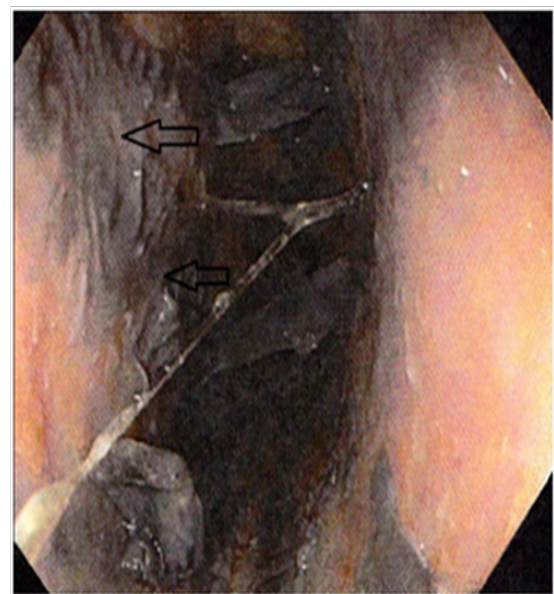

Figure I Endoscopic view of the middle esophagus revealing black mucosa with friable tissue.

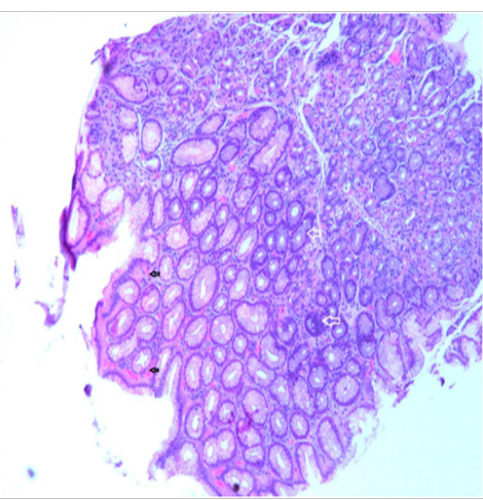

Figure 2 Esophageal biopsy (H\&E, 20x) showed demarcation line in the upper submucosa with a predominance of neutrophilic granulocytes and scattered macrophages along with platelet thrombus in the small submucosal vessel.

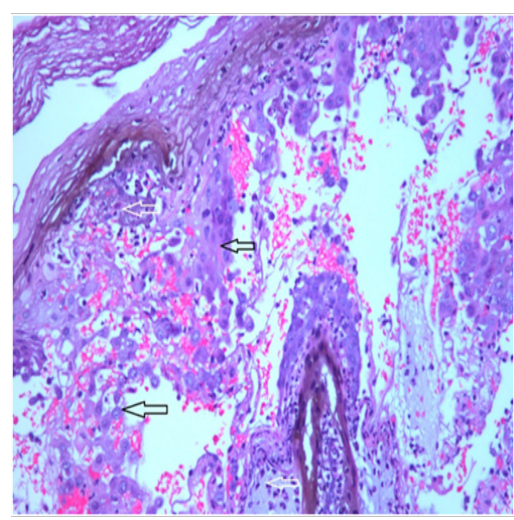

Figure 3 Esophageal biopsy (H\&E, 40x) showed demarcation line in the upper submucosa with a predominance of neutrophilic granulocytes and scattered macrophages along with platelet thrombus in the small submucosal vessel

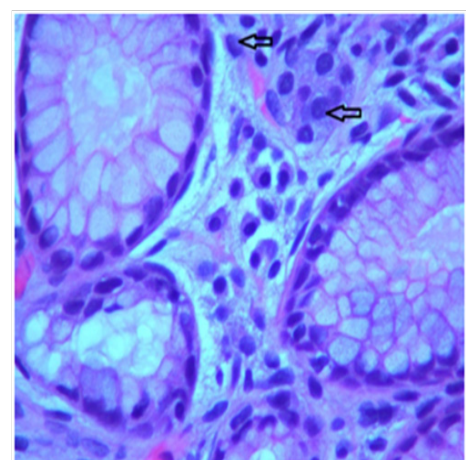

Figure 4 Recto-sigmoidal biopsy showed (H\&E, 400x) showed Infiltrating adenocarcinoma.

\section{Discussion}

The black esophagus (BE) or acute esophagus necrosis (AEN) is one of rare but fatal disease entity of Gurvits syndrome. The mean age is 44years with male being common. The criteria to diagnose BE are circumferential diffuse black esophagus with/without exudates on endoscopy; involvement lower one-third of esophagus ended abruptly at GE (gastroesophageal) junction; and without caustic ingestion. ${ }^{4}$

The presentation varies from asymptomatic to bleeding diathesis: hematemesis and melena in $90 \%$ of the cases. General examination may be significant if associated with cachexia, fever, hemodynamic instabilities or complications. Complications are esophagus stenosis or strictures (10.8\%), perforation (6.8\%) and spreading of inflammation and infection to the mediastinum (5.9\%). A strong association between AEN and DKA (diabetic ketoacidosis) has reported in 4cases of BE in 29 hospital admissions. ${ }^{5}$

Diagnosis is confirmed with gastroscopy .Though biopsy is recommended, it is not required for diagnosis purpose. Histology findings include necrosis of mucosa, submucosa extending to muscularis propia, wide spread necrotic debris and leucocyte infiltrates inflammatory changes in muscles, visible vascular thrombi on endoscopy.

Various differential diagnoses such as melanosis esophagi, melanoma, acanthosis nigricans, herpes simplex esophagitis, candida esophagitis, and lactobacillus acidophilus esophagitis, ingestion of medications or corrosive agents should take into consideration.

In our patient, in addition to underlying infiltrative colonic adenocarcinoma and rapid weight loss from cachectic condition, disruption of mucosa barriers from anemia, provoked thromboembolic phenomenon-portal vein thrombosis predisposes to the black esophagus.

AEN is solely managed with fluid resuscitation, IV PPI (proton pump inhibitors) ${ }^{6}$ with targeted underlying comorbidities management while surgery is sole option for cases with complication. Black esophagus is rare but increasing incidental endoscopic finding in older male populations with upper GI symptoms. Management should emphasize on improving perfusion, treating comorbidities and associated infection if present, and reduce further tissue insult using proton pump inhibitors to prevent complications. Generally, black esophagus follows an indolent course and has an expected endoscopic and histological trajectory. The mortality rate is high in setting of underlying comorbidities and diseases. 


\section{Acknowledgements}

None.

\section{Conflict of interest}

The authors declare no conflict of interest.

\section{References}

1. Gurvits GE, Shapsis A, Lau N, et al. Acute esophageal necrosis: a rare syndrome. Journal of gastroenterology. 2007;42(1):29-38.

2. Singh D, Singh R, Laya AS. Acute esophageal necrosis: a case series of five patients presenting with Black esophagus. Indian J Gastroenterol. 2011;30(1):41-45.
3. Goldenberg SP, Wain SL, Marignani P. Acute necrotizing esophagitis. Gastroenterology. 1990;98(2):493-496.

4. Moretó M, Ojembarrena E, Zaballa M, et al. Idiopathic acute esophageal necrosis: not necessarily a terminal event. Endoscopy. 1993;25(8):534 538 .

5. Kim YH, Choi SY. Black esophagus with concomitant candidiasis developed after diabetic ketoacidosis. World journal of gastroenterology:WJG. 2007;1413(42):5662-5663.

6. Burtally A, Gregoire P. Acute esophageal necrosis and low-flow state. Canadian Journal of Gastroenterology and Hepatology. 2007;21(4):245-247. 